\title{
Comparative Study of Vitamin D Level between Psoriatic Patients and Psoriatic Arthritis Patients
}

\author{
Abd Elmegeed A. El-Ashmawy ${ }^{1}$, Abd Alshafy A. Haseeb ${ }^{2}$, Hatem G. Abd_Allah ${ }^{3}$, Hesham S. \\ Abd-Alsamie ${ }^{4}$, Mostafa A. Elhelaly ${ }^{5}$ \\ ${ }^{1,2,3}$ Department of Physical Medicine, Rheumatology and Rehabilitation, Faculty of Medicine, 4Department of clinical \\ pathology, Damietta Faculty of Medicine, Al-Azhar University, ${ }^{5}$ Department of Physical Medicine, Rheumatology and \\ Rehabilitation, Faculty of Medicine, Al-Azhar University, Damietta, Egypt.
}

Corresponding author: Mostafa A. Elhelaly; Mobile: 01008828261; Email: dr.mostafa9192@ yahoo.com

\begin{abstract}
Background Psoriatic arthritis (PsA) is a seronegative (rheumatoid factor-negative) usually Cyclic Citrullinated Peptide (CCP) antibody negative, immunologically triggered, chronic inflammatory joint disease within the framework of psoriasis. Psoriasis affects $2-4 \%$ of the general population. It is a common, chronic, relapsing/remitting, immune-mediated systemic disease characterized by skin lesions including red, scaly patches, papules and plaques, which usually itch. Vitamin D is actually a fat-soluble steroid prohormone that has endocrine, paracrine and autocrine functions.

Aim of the Work: To investigate the level of serum vitamin D in patients with psoriatic arthritis and compare it with patients with psoriasis to show if there is a role of vitamin D in development of arthritis in psoriatic patients or not.

Patients and Methods: This study is a cross sectional study in which fifty patients with psoriatic arthritis and psoriasis age range 16-50 years selected from those attending the outpatient clinic and inpatients of rheumatology, physical medicine \&rehabilitation department, Al-Azhar university hospitals from 2017 to 2018 and divided into two groups: Group (A): (25) patients with psoriatic arthritis were diagnosed according to the criteria for psoriatic arthritis classification "CASPAR criteria". Group (B): (25) patients with skin psoriasis. In this study we measured of 25 hydroxy vitamin D3 using commercial ELISA kits, ESR, CRP, CBC, Blood urea, serum creatinine, Liver enzymes (SGOT \& SGPT), Serum uric acid, Fasting blood glucose, RF, urine analysis, ANA, HCV Ab and HIV Ab. Plain x-ray on both hands postero-anterior view, Plain x-ray on lumbosacral spine antero- posterior and lateral views and Plain x-ray on both sacroiliac joints "cone" view.

Results: There was female sex predicliction in psoriatic arthritis group. Otherwise, both groups were comparable regarding age, BMI, residence, co-morbid diseases, disease characteristics, and laboratory investigations, except significant increase of WBCs and inflammatory markers (ESR and CRP) in psoriatic arthritis group. Diseases severity was mild in $72 \%$, moderate in $22 \%$ and severe in $6.0 \%$; and there was statistically significant increase of moderate and severe disease in PA when compared to psoriasis group. vitamin D was sufficient in 32.0\%, insufficient in 34.0\% and deficient in 34.0\%; and there was no significant difference between psoriasis and PA groups. In addition, vitamin D levels were increased in psoriasis when compared to PA groups. However, the difference was statistically non-significant. No significant relation was found between vitamin D from one side and each of patient characteristics, laboratory investigations (except significant positive correlation with hemoglobin and RBCs), disease characteristics (except significant inverse (negative) correlation with disease duration and disease severity), X-ray findings, and psoriatic arthritis disease activity indices.

Conclusion: Vitamin D deficiency/insufficiency was common among psoriasis and psoriatic arthritis patients and the deficiency was significantly and inversely correlated with disease severity (PASI) and disease duration. Thus, is thought to play a role in development (or a consequence) of psoriasis. On the other hand, there was no correlation with PA disease activity and severity indices. Thus, the association seems to be linked to psoriasis proper. Vitamin D supplementation thought to have a potential therapeutic role in treatment of psoriasis.
\end{abstract}

Keywords: Psoriatic Arthritis, Psoriasis, Vitamin D.

\section{INTRODUCTION}

Psoriatic arthritis (PsA) is a seronegative (rheumatoid factor-negative) usually Cyclic Citrullinated Peptide (CCP) antibody negative, immunologically triggered, chronic inflammatory joint disease within the framework of psoriasis. PsA has many similarities to rheumatoid arthritis and other inflammatory joint diseases making the diagnosis more difficult. About one-fourth to one- third of all psoriasis patients (depending on study design and population studied) develop musculoskeletal involvement typical for this disease. In about $20 \%$ of patients inflammatory joint disease appears usually many years after the manifestation of skin disease. PsA can appear without or before the manifestation of cutaneous signs and symptoms. The male:female ratio is about $1: 1.3^{(1)}$. 
Psoriasis affects 2-4\% of the general population. It is a common, chronic, relapsing/ remitting, immune-mediated systemic disease characterized by skin lesions including red, scaly patches, papules and plaques, which usually itch ${ }^{(2)}$.

Vitamin D is actually a fat-soluble steroid prohormone that has endocrine, paracrine and autocrine functions. The endocrine effects of vitamin $\mathrm{D}$ are mainly involved in serum calcium homeostasis. The paracrine and autocrine effects of vitamin D depend on genetic transcription, unique to the type of cell expressing nuclear vitamin D receptors. These potential effects include inhibition of cell proliferation, promotion of cell differentiation, and apoptosis which may in turn have roles in cancer, immunity and many organ systems ${ }^{(3)}$.

Autoimmune diseases are characterized by a loss of immune homeostasis resulting in corrupted self-antigen recognition followed by the destruction of body tissue by autoreactive immune cells. A combination of genetic predisposition, epidemiological and environmental risk factors contributes to the development of autoimmune diseases ${ }^{(4)}$.

Compromised vitamin D status has been associated with an increased risk for Th1 cytokinemediated autoimmune diseases, including insulin dependent diabetes mellitus (IDDM), multiple sclerosis (MS), inflammatory bowel disease and rheumatoid arthritis (RA) ${ }^{(5)}$.

\section{AIM OF THE WORK}

The aim of this study is to investigate the level of serum vitamin $D$ in patients with psoriatic arthritis and compare it with patients with psoriasis to show if there is a role of vitamin $D$ in development of arthritis in psoriatic patients or not.

\section{PATIENTS AND METHODS}

This study is a cross sectional study in which fifty patients with psoriatic arthritis and psoriasis age range 16-50 years selected from those attending the outpatient clinic and inpatients of rheumatology, physical medicine \&rehabilitation department, Al-Azhar university hospitals from 2017 to 2018 and divided into two groups: 1(Group A): (25) patients with psoriatic arthritis were diagnosed according to the criteria for psoriatic arthritis classification "CASPAR criteria". 2(Group B): (25) patients with skin psoriasis.

An informed consent was taken by each patient before the procedure. The study was

\author{
approved by the Ethics Board of Al-Azhar \\ University.
}

\section{Patient's selection \\ Inclusion criteria:}

1-Patients with psoriatic arthritis diagnosed as CASPAR criteria which include: Evidence of psoriasis (current, past or family): two points if current history of psoriasis, one point others. Psoriatic nail dystrophy: one point. Negative rheumatoid factor: one point. Dactylitis (current or past history): one point. Radiographic evidence of juxtaarticular new bone formation: one point. Three or more points have $99 \%$ specificity and 92\% sensitivity for diagnosis of psoriatic arthritis.

2-Patients with psoriasis symptomatized from: Red, raised and inflamed patches of skin. Silver-white scales or plaques on the red patches. Dry skin that may crack and bleed. Soreness around patches. Itching and burning sensations around patches. Thick and pitted nails.

\section{Exclusion criteria:}

1-Other forms of inflammatory arthritis as Rheumatoid Arthritis and the other seronegative spondyloarthropathies (Ankylosing Spondylitis, Reactive Arthritis and Inflammatory bowel disease associated Arthritis) particularly when patients have sacroiliitis and enthesitis.

2-Patients with other chronic diseases which might have relation with vitamin D deficiency (as hepatic and renal disease or failure).

3-Patients with drugs which might have relation with vitamin $\mathrm{D}$ deficiency (as anti epileptic drugs as carbamazepine, rafimpicin, isoniazid, anti fungal drugs as ketoconazole and steroids).

4-HCV and HIV patients.

5-Pregnancy and lactating women.

\section{Methods:}

For every patient included in the study, the following items were fulfilled:

Detailed history including: Personal history: Name, age, sex, residence, occupation, family history, marital status, drug intake, associated faulty habits or life style, precipitating factors, seasonal variation, presence of any systemic disease.

Present history: Onset, course, duration. 
General physical examination to determine any other systemic disease that may be related to condition.

Complete Locomotor and dermatological examinationto detect the lesions and the distribution all over the body.

Laboratory investigations including measurement of 25 hydroxy vitamin D3 using commercial ELISA kits. ESR: using western green tubes method. CRP using latex agglutination test. Complete blood count : using automated cell counter. Blood urea, serum creatinine, Liver enzymes (SGOT \& SGPT), Serum uric acid and Fasting blood glucose by spectrophometeric method. Rheumatoid factor using latex agglutination test. Complete urine analysis by microscopic examination. ANA by ELISA.

\section{HCV abs and HIV by ELISA.}

Radiology: Plain $\mathrm{x}$-ray on both hands postero-anterior view. Plain $\mathrm{x}$-ray on lumbosacral spine antero- posterior and lateral views. Plain $x-$ ray on both sacroiliac joints "cone" view.

Statistical analysis: All data were collected, tabulated and statistically analyzed using SPSS 22.0 for windows (SPSS Inc., Chicago, IL, USA) \& MedCalc 13 for windows (MedCalc Software bvba, Ostend, Belgium).

Continuous Quantitative variables e.g. age were expressed as the mean $\pm \mathrm{SD} \&$ median (range), and categorical qualitative variables were expressed as absolute frequencies (number) \& relative frequencies (percentage).

Continuous data were checked for normality by using Shapiro Walk test. Independent samples Student's t-test was used to compare two groups of normally distributed data while MannWhitney $U$ test was used for non-normally distributed data. One Way ANOVA test was used to compare more than two groups of normally distributed data while Kraskall Wallis $\mathrm{H}$ test was used for non-normally distributed data. Categorical data were compared using Chi-square test or Fisher's exact test when appropriate.

Spearman's rank correlation coefficient was calculated to assess correlations between study parameters. We consider (+) sign as indication for direct correleation i.e. \& (-) sign as indication for inverse correleation, also we consider values near to 1 as strong correleation \& values near 0 as weak correleation.
All tests were two sided. p-value $<0.05$ was considered statistically significant, p-value < 0.001 was considered highly statistically significant, and $\mathrm{p}$-value $\geq 0.05$ was considered non statistically significant.

\section{RESULTS}

Table (1): Patient characteristics of studied populations.

\begin{tabular}{|c|c|c|c|c|c|}
\hline \multicolumn{2}{|c|}{ Variable } & Psoriasis & Psoriatic A & Total & $P$ value \\
\hline \multicolumn{2}{|c|}{ Age (years) } & $31.60 \pm 8.25$ & $31.96 \pm 8.66$ & $\begin{array}{c}31.78 \pm 8.37 \\
18-52\end{array}$ & 0.88 (ns) \\
\hline \multirow{2}{*}{ Sex } & Male & $17(68.0 \%)$ & $9(36.0 \%)$ & $26(52.0 \%)$ & \multirow{2}{*}{ 0.024* } \\
\hline & Female & $8(32.0 \%)$ & $16(64.0 \%)$ & $24(48.0 \%)$ & \\
\hline \multicolumn{2}{|c|}{ BMI(kg/m2) } & $26.74 \pm 1.52$ & $27.00 \pm 1.11$ & $\begin{array}{c}26.87 \pm 1.32 \\
23.62-30.07\end{array}$ & 0.49 (ns) \\
\hline \multirow{2}{*}{ Residence } & Rural & $15(60.0 \%)$ & $13(52.0 \%)$ & $28(56.0 \%)$ & \multirow{2}{*}{$0.38(\mathrm{~ns})$} \\
\hline & Urban & $10(40.0 \%)$ & $12(48.0 \%)$ & $22(44.0 \%)$ & \\
\hline \multicolumn{2}{|l|}{ Smoking } & $5(20.0 \%)$ & $7(28.0 \%)$ & $12(24.0 \%)$ & $0.37(\mathrm{~ns})$ \\
\hline \multicolumn{2}{|c|}{ Hypertension } & $5(20.0 \%)$ & $9(36.0 \%)$ & $14(28.0 \%)$ & $0.17(\mathrm{~ns})$ \\
\hline
\end{tabular}

In the present work, patient age ranged from 18 to 52 years, and there was no significant difference between psoriasis and PA groups. Patient gender distribution revealed that, $52.0 \%$ were males and $48 \%$ were females, and there was significant increase of females in psoriatic arthritis group when compared to psoriasis group (64.0\% vs $32.0 \%$ respectively). However, there was no significant difference between both groups as regard to BMI, residence, smoking and hypertension.

Table (2): Clinical data of studied populations.

\begin{tabular}{|c|c|c|c|c|c|c|c|c|}
\hline & \multicolumn{2}{|c|}{ Psoriasis } & \multicolumn{2}{|c|}{ PA } & \multicolumn{2}{|c|}{ Total } & \multirow{2}{*}{$\begin{array}{c}P \\
\text { value }\end{array}$} \\
\hline & & $\mathbf{N}$ & $\%$ & $\mathbf{N}$ & $\%$ & $\mathbf{N}$ & $\%$ & \\
\hline \multirow{3}{*}{$\begin{array}{l}\text { Disease } \\
\text { duration }\end{array}$} & $<5$ years & 15 & $60.0 \%$ & 12 & $48.0 \%$ & 27 & $54.0 \%$ & \multirow{3}{*}{$\begin{array}{l}0.56 \\
(\mathrm{~ns})\end{array}$} \\
\hline & $5-10$ years & 6 & $24.0 \%$ & 6 & $24.0 \%$ & 12 & $24.0 \%$ & \\
\hline & $>10$ years & 4 & $16.0 \%$ & 7 & $28.0 \%$ & 11 & $22.0 \%$ & \\
\hline \multirow{2}{*}{ Onset } & Sudden & 12 & $48.0 \%$ & 15 & $60.0 \%$ & 27 & $54.0 \%$ & \multirow{2}{*}{$\begin{array}{l}0.28 \\
(\mathrm{~ns})\end{array}$} \\
\hline & Gradual & 13 & $52.0 \%$ & 10 & $40.0 \%$ & 23 & $46.0 \%$ & \\
\hline \multirow{4}{*}{ Type } & Plaque & 19 & $76.0 \%$ & 19 & $76.0 \%$ & 38 & $76.0 \%$ & \multirow{4}{*}{$\begin{array}{l}0.92 \\
(\mathrm{~ns})\end{array}$} \\
\hline & Guttate & 4 & $16.0 \%$ & 3 & $12.0 \%$ & 7 & $14.0 \%$ & \\
\hline & Inverse & 1 & $4.0 \%$ & 1 & $4.0 \%$ & 2 & $4.0 \%$ & \\
\hline & Pustular & 1 & $4.0 \%$ & 2 & $8.0 \%$ & 3 & $6.0 \%$ & \\
\hline \multirow{3}{*}{ Course } & Progressive & 16 & $64.0 \%$ & 19 & $76.0 \%$ & 35 & $70.0 \%$ & \multirow{3}{*}{$\begin{array}{l}0.31 \\
\text { (NS) }\end{array}$} \\
\hline & stationary & 7 & $28.0 \%$ & 6 & $24.0 \%$ & 13 & $26.0 \%$ & \\
\hline & Regressive & 2 & $8.0 \%$ & 0 & $.0 \%$ & 2 & $4.0 \%$ & \\
\hline \multirow{2}{*}{$\begin{array}{l}\text { Family } \\
\text { history }\end{array}$} & Positive & 6 & $24.0 \%$ & 7 & $28.0 \%$ & 13 & $26.0 \%$ & \multirow{2}{*}{$\begin{array}{l}0.50 \\
\text { (ns) }\end{array}$} \\
\hline & Negative & 19 & $76.0 \%$ & 18 & $72.0 \%$ & 37 & $74.0 \%$ & \\
\hline \multirow{2}{*}{\begin{tabular}{|l} 
Nail \\
affection
\end{tabular}} & Positive & 5 & $20.0 \%$ & 10 & $40.0 \%$ & 15 & $30.0 \%$ & \multirow{2}{*}{$\begin{array}{l}0.11 \\
(\mathrm{~ns})\end{array}$} \\
\hline & Negative & 20 & $80.0 \%$ & 15 & $60.0 \%$ & 35 & $70.0 \%$ & \\
\hline \multirow{2}{*}{ Arthritis } & Oligoarticular & & & 14 & $56.0 \%$ & & & \\
\hline & Polyarticular & & & 11 & $44.0 \%$ & & & \\
\hline
\end{tabular}

In the present work, disease duration was less than 5 years in 54\%, from 5- 10 years in $24 \%$ and more than 10 years in $22.0 \%$; the disease onset was sudden in $54 \%$ and gradual in $46.0 \%$; the plaque type was reported in $76 \%$, guttate in $14 \%$, inverse in $4.0 \%$ and pustular in $6.0 \%$; the course was progressive in $70.0 \%$, stationary in $26 \%$ and regressive in $4.0 \%$; the family history was positive 
in $26 \%$; nails were affected in $30.0 \%$ and psoriatic arthritis was oligoarticular in $56 \%$ and polyarticular in $44 \%$.

Table (3): DIP joint in hand X-ray in psoriatic arthritis.

\begin{tabular}{|l|c|c|}
\hline & $\mathbf{N}$ & $\mathbf{\%}$ \\
\hline Narrowing & 10 & 40.0 \\
\hline Sclerosis & 6 & 24.0 \\
\hline Erosion & 6 & 24.0 \\
\hline Periostitis & 3 & 12.0 \\
\hline Total & 25 & 100.0 \\
\hline
\end{tabular}

Hand- $\mathrm{x}$ ray examination revealed narrowing in $40 \%$, sclerosis in $24 \%$, erosion in $24 \%$ and periostitis in $12 \%$ of DIP joint of those with psoriatic arthritis.

Table (4): Sacroiliac X ray in psoriatic arthritis.

\begin{tabular}{|l|c|c|}
\hline & N & \% \\
\hline Normal & 19 & 76.0 \\
\hline Blurring & 2 & 8.0 \\
\hline Pseudowidening & 2 & 8.0 \\
\hline Sclerosis & 1 & 4.0 \\
\hline Ankyloses & 1 & 4.0 \\
\hline Total & 25 & 100.0 \\
\hline
\end{tabular}

Sacroiliac joint examination by X-ray revealed was normal in $76 \%$ of patients, showed blurring in $8 \%$, pseudowidening in $8.0 \%$, sclerosis in $4.0 \%$ and ankyloses in $4.0 \%$.

Table (5): Laboratory data of studied populations.

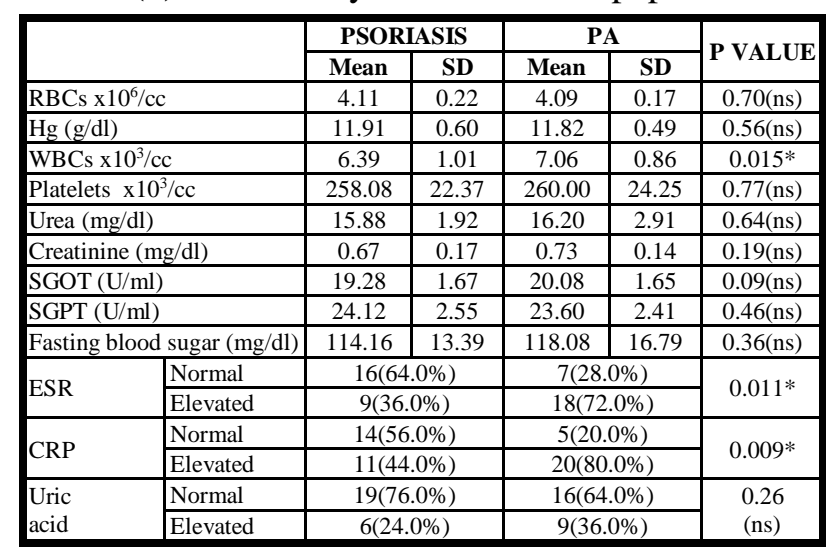

In the present work, there was no significant difference between psoriasis and psoriatic arthritis as regard to laboratory data, except significant decrease of WBCs in psoriasis when compared to psoriatic arthritis $(6.39 \pm 1.01 \mathrm{vs}$ $7.06 \pm 0.86$ respectively).

In the present study, there was statistically significant increase of elevated ESR, elevated CRP in psoriasis arthritis group when compared to psoriasis group $(72.0 \%, 80.0 \%$, vs $36.0 \%, 44.0 \%$ and respectively); while there was no significant difference between both groups as regard to serum uric acid.

Table (6): Psoraitic disease severity in studied populations.

\begin{tabular}{|c|c|c|c|c|c|c|c|c|}
\hline & \multicolumn{4}{|c|}{ Group } & \multirow{2}{*}{\multicolumn{2}{|c|}{ Total }} & \multirow{3}{*}{$\begin{array}{c}P \\
\text { value }\end{array}$} \\
\hline & & \multicolumn{2}{|c|}{ Psoriasis } & \multicolumn{2}{|r|}{$\overline{\mathbf{P A}}$} & & & \\
\hline & & $\mathbf{n}$ & $\%$ & $\mathbf{n}$ & $\%$ & $\mathbf{n}$ & $\%$ & \\
\hline \multirow{3}{*}{ Severity } & Mild & 21 & $84.0 \%$ & 15 & $60.0 \%$ & 36 & $72.0 \%$ & \multirow{3}{*}{$0.050^{*}$} \\
\hline & Moderate & 4 & $16.0 \%$ & 7 & $28.0 \%$ & 11 & $22.0 \%$ & \\
\hline & Severe & 0 & $0.0 \%$ & 3 & $12.0 \%$ & 3 & $6.0 \%$ & \\
\hline \multicolumn{2}{|c|}{ PASI (mean \pm SD) } & \multicolumn{2}{|c|}{$7.61 \pm 2.79$} & \multicolumn{2}{|c|}{$10.73 \pm 5.28$} & \multicolumn{2}{|c|}{$9.17 \pm 4.47$} & $0.012^{*}$ \\
\hline
\end{tabular}

As regard to disease severity, it was mild in $72 \%$, moderate in $22 \%$ and severe in $6.0 \%$; and there was statistically significant increase of moderate and severe disease in PA when compared to psoriasis group (28.0\%, $12.0 \%$ vs $16.0 \%$ and $0.0 \%$ respectively). In addition, the mean PASI score was significantly increased in PA when compared to psoriasis group (10.73 \pm 5.28 vs $7.61 \pm 2.79$ respectively).

Table (7): Vitamin D in studied populations.

\begin{tabular}{|c|c|c|c|c|c|c|c|c|}
\hline & & \multicolumn{4}{|c|}{ Group } & \multirow{2}{*}{\multicolumn{2}{|c|}{ Total }} & \multirow{3}{*}{$P$ value } \\
\hline & & \multicolumn{2}{|c|}{ Psoriasis } & \multicolumn{2}{|c|}{$\mathbf{P A}$} & & & \\
\hline & & $\mathbf{N}$ & $\%$ & $\mathbf{N}$ & $\%$ & $\mathbf{N}$ & $\%$ & \\
\hline \multirow{3}{*}{$\begin{array}{l}\text { Vitamin } \\
\text { D }\end{array}$} & Sufficient & 10 & $40.0 \%$ & 6 & $24.0 \%$ & 16 & $32.0 \%$ & \multirow{3}{*}{$\begin{array}{l}0.28 \\
(\mathrm{~ns})\end{array}$} \\
\hline & \begin{tabular}{|l} 
Insufficient \\
\end{tabular} & 9 & $36.0 \%$ & 8 & $32.0 \%$ & 17 & $34.0 \%$ & \\
\hline & Deficient & 6 & $24.0 \%$ & 11 & $44.0 \%$ & 17 & $34.0 \%$ & \\
\hline \multicolumn{2}{|c|}{ Vitamin D } & \multicolumn{2}{|c|}{$26.11 \pm 5.97$} & \multicolumn{2}{|c|}{$22.83 \pm 8.40$} & \multicolumn{2}{|c|}{$24.47 \pm 7.40$} & $0.12(\mathrm{~ns})$ \\
\hline
\end{tabular}

As regard to vitamin $\mathrm{D}$, it was sufficient in $32.0 \%$, insufficient in $34.0 \%$ and deficient in $34.0 \%$; and there was no significant difference between psoriasis and PA groups. In addition, vitamin D levels were increased in psoriasis when compared to PA groups. However, the difference was statistically non-significant.

Table (8): Psoriatic arthritis severity indices.

\begin{tabular}{|l|c|c|c|c|}
\hline & Mean & SD & Minimum & Maximum \\
\hline BASDAI & 2.48 & 0.51 & 2.00 & 3.00 \\
\hline BASFI & 2.60 & 0.50 & 2.00 & 3.00 \\
\hline BASMI & 1.64 & 0.56 & 1.00 & 3.00 \\
\hline
\end{tabular}

As regard to BASDAI, it was ranged from 2 to 3 , the mean value was $2.48 \pm 0.51$; while BASFI ranged from 2 to 3 , the mean value was $2.60 \pm 0.50$ and BASMI ranged from 1 to 3 , the mean value was $1.64 \pm 0.56$.

Table (9): Vitamin D in relation to age, BMI, and laboratory data.

\begin{tabular}{|l|c|c|}
\hline \multirow{2}{*}{} & \multicolumn{2}{|c|}{ Vitamin D } \\
\cline { 2 - 3 } & $\mathbf{r}$ & $\mathbf{p}$ \\
\hline Age & -0.06 & 0.67 \\
\hline BMI & -0.07 & 0.62 \\
\hline RBCs & 0.29 & $0.038^{*}$ \\
\hline Hemoglobin & 0.28 & $0.48^{*}$ \\
\hline WBCs & -0.07 & 0.62 \\
\hline Platelets & -0.09 & 0.52 \\
\hline Urea & -0.17 & 0.22 \\
\hline
\end{tabular}




\begin{tabular}{|l|c|c|}
\hline Creatinine & -0.14 & 0.30 \\
\hline SGOT & 0.05 & 0.71 \\
\hline SGPT & 0.25 & 0.07 \\
\hline Fasting blood glucose & 0.03 & 0.83 \\
\hline
\end{tabular}

In the present study, there was proportional (positive), statistically significant, moderate correlation between vitamin $\mathrm{d}$ and each of RBCs and hemoglobin. Otherwise, there was no significant difference between vitamin $\mathrm{D}$ and age, BMI, WBCs, platelets, urea, creatinine, SGOT, SGPT and fasting blood glucose.

Table (10): VitaminD in relation to clinical data

\begin{tabular}{|l|l|c|c|c|c|c|}
\hline \multicolumn{2}{|c|}{} & Mean & SD & Mini. & Maxi. & P value \\
\hline \multirow{2}{*}{ Disease } & $<5$ years & 27.17 & 6.75 & 13.10 & 36.20 & \\
duration & 5-10 years & 22.62 & 6.81 & 8.40 & 31.90 & \multirow{2}{*}{$0.011^{*}$} \\
& $>10$ years & 19.86 & 7.18 & 9.40 & 35.20 & \\
\hline \multirow{2}{*}{ Onset } & Sudden & 24.35 & 6.93 & 8.40 & 35.20 & 0.90 \\
& Gradual & 24.61 & 8.07 & 9.40 & 36.20 & $(\mathrm{~ns})$ \\
\hline \multirow{5}{*}{ Type } & Plaque & 23.54 & 7.39 & 8.40 & 36.20 & \\
& Guttate & 28.90 & 5.84 & 17.90 & 34.10 & \multirow{2}{0}{$0.37(\mathrm{~ns})$} \\
& Inverse & 25.45 & 10.53 & 18.00 & 32.90 & \\
& Pustular & 25.30 & 9.24 & 16.90 & 35.20 & \\
\hline \multirow{3}{*}{ Course } & Progressive & 24.12 & 7.75 & 8.40 & 36.20 & \multirow{2}{*}{0.36} \\
& stationary & 24.28 & 6.64 & 14.10 & 33.90 & $(\mathrm{~ns})$ \\
\hline Family & Regressive & 31.80 & 0.14 & 31.70 & 31.90 & \\
History & Positive & 24.45 & 8.72 & 9.40 & 35.10 & 0.99 \\
\hline Nail & Negative & 24.48 & 7.01 & 8.40 & 36.20 & (ns) \\
affection & Positive & 24.54 & 7.33 & 14.10 & 36.20 & 0.96 \\
& Negative & 24.44 & 7.54 & 8.40 & 35.20 & (ns) \\
\hline
\end{tabular}

In the present study, there was statistically significant decrease of vitamin $\mathrm{D}$ with increased disease duration (inverse correlation). However, there was no significant relation between vitamin $\mathrm{D}$ and disease onset type, course, family history or nail affection.

Table (11): Vitamin D in relation to type of arthritis, and X-ray in PA

\begin{tabular}{|l|l|c|c|c|c|c|}
\hline \multicolumn{2}{|l|}{} & Mean & SD & Mini. & Maxi. & p \\
\hline Type of & Oligoarticular & 25.46 & 7.28 & 15.80 & 36.20 & \multirow{2}{*}{0.08} \\
\hline \multirow{3}{*}{ Hand } & Polyarticular & 19.47 & 8.85 & 8.40 & 34.10 & \\
X-ray & Narrowing & 22.37 & 8.04 & 9.40 & 35.20 & \\
& Sclerosis & 21.88 & 11.01 & 8.40 & 35.10 & \multirow{2}{*}{0.86} \\
& Erosion & 25.45 & 8.76 & 15.20 & 36.20 & \\
& Periostitis & 21.00 & 5.63 & 17.50 & 27.50 & \\
Sacroiliac & Normal & 23.71 & 8.76 & 9.40 & 36.20 & \\
X-ray & Blurring & 16.08 & 10.86 & 8.40 & 23.76 & \\
& Pseudowidening & 24.20 & 1.41 & 23.20 & 25.20 & \multirow{2}{*}{0.63} \\
& Sclerosis & 25.50 & & 25.50 & 25.50 & \\
& Ankylosis & 14.10 & & 14.10 & 14.10 & \\
\hline
\end{tabular}
significant relation between vitamin $\mathrm{D}$ from one side and each of type of arthritis, Hand x-ray findings and sacroiliac $\mathrm{x}$ ray findings.

Table (12): Vitamin D in relation to psoriatic disease severity.

\begin{tabular}{|l|c|c|c|c|c|}
\hline & Mean & S D & Minimum & Maximum & P value \\
\hline Mild & 26.06 & 6.67 & 8.40 & 35.20 & \\
\cline { 1 - 5 } Moderate & 21.49 & 8.54 & 9.40 & 36.20 & \multirow{2}{*}{$0.026^{*}$} \\
\hline Severe & 16.33 & 2.01 & 14.10 & 18.00 & \\
\hline
\end{tabular}

\begin{tabular}{|l|l|l|l|l|l|}
\hline Total & 24.47 & 7.40 & 8.40 & 36.20 & \\
\hline
\end{tabular}

In the present study, there was statistically significant decrease of vitamin D levels with increased disease severity.

Table (13): Vitamin D in relation to ESR, CRP and uric acid.

\begin{tabular}{|c|l|c|c|c|c|c|}
\hline \multicolumn{2}{|c|}{} & Mean & SD & Minimum & Maximum & $\begin{array}{c}\text { P } \\
\text { value }\end{array}$ \\
\hline \multirow{2}{*}{ ESR } & Normal & 25.08 & 7.43 & 8.40 & 35.20 & \multirow{2}{*}{0.59} \\
\cline { 2 - 6 } & Elevated & 23.94 & 7.47 & 9.40 & 36.20 & \\
\hline \multirow{2}{*}{ CRP } & Normal & 25.57 & 6.98 & 13.10 & 35.20 & \multirow{2}{*}{0.41} \\
\cline { 2 - 6 } & Elevated & 23.80 & 7.68 & 8.40 & 36.20 & \\
\hline \multirow{2}{*}{$\begin{array}{l}\text { Uric } \\
\text { acid }\end{array}$} & Normal & 24.82 & 7.52 & 8.40 & 35.20 & \multirow{2}{*}{0.61} \\
\cline { 2 - 6 } & Elevated & 23.65 & 7.30 & 9.40 & 36.20 & \\
\hline
\end{tabular}

In the present study, there was no significant relation between vitamin $\mathrm{D}$ and each of ESR, CRP and uric acid.

Table (14): Correlation between vitamin D and psoriatic arthritis severity indices.

\begin{tabular}{|l|c|c|}
\hline \multirow{2}{*}{} & \multicolumn{2}{|c|}{ Vitamin D } \\
\cline { 2 - 3 } & $\mathbf{r}$ & $\mathbf{P}$ \\
\hline BASDAI & -0.19 & 0.36 \\
\hline BASFI & -0.17 & 0.41 \\
\hline BASMI & -0.13 & 0.51 \\
\hline
\end{tabular}

In the present work, there was no significant relation between vitamin $\mathrm{D}$ and each of BASDAI, BASFI and BASMI.

\section{DISCUSSION}

In the present work, patient age ranged from 18 to 52 years, and there was no significant difference between psoriasis and PA groups (it was $31.60 \pm 8.25$ and $31.96 \pm 8.66$ in psoriasis and PA groups respectively. Patient gender distribution revealed that, $52.0 \%$ were males and $48 \%$ were females, and there was significant increase of females in psoriatic arthritis group when compared to psoriasis group (64.0\% vs $32.0 \%$ respectively).

Results of the present work are comparable to those reported by Ibrahim et $\boldsymbol{a l} .{ }^{(6)}$ who included thirty psoriatic arthritis patients (21female,9 male) with age range from 17-67 years (mean age 35.03 \pm 15.56 ).

In addition, resutls of the present work regarding PA patients are in agreement with Braun et $\boldsymbol{a l} .{ }^{(7)}$ who found that most of patients were females.

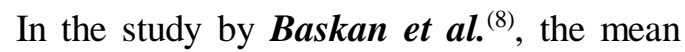
age of the 52 PsA patients (23 males, 29 females) was $41.9 \pm 8.4$ years. This age is higher than the present work and this reflects the early development of disease. However, the sex 
distribution is comparable between both studies, as there was increase of female sex predilection in psoriatic arthritis group.

In the present work, the disease duration in PA group was $<5$ years in 48\%, 5-10 years in 24\% and $>10$ years in $28 \%$, while in psoriasis group $60 \%$ had disease duration $<5$ years, $24 \%$ had duration 510 years and $16 \%$ had duration $>10$ years with no signficant differnce between both groups. These results are comparable to that reproted by Ibrahim et $\boldsymbol{a l} .{ }^{(6)}$ who reported that, in psoriatic arthritis, $46.7 \%$ of selected patient below 5 years duration of disease while $30 \%$ of selected patient from 5-10 years and $23.3 \%$ of selected patient above 10 years.

In addition, Allayali et al $^{\left({ }^{(9)}\right.}$ from Saudi Arabia reported that, the average years for patients having psoriasis was 7.75 years

In the present work, the plaque type was reported in $76 \%$, guttate in $14 \%$, inverse in $4.0 \%$ and pustular in $6.0 \%$. The disease onset was sudden in $54 \%$ and gradual in $46.0 \%$; the family history was positive in $26 \%$; nails were affected in $30.0 \%$ and psoriatic arthritis was oligoarticular in $56 \%$ and polyarticular in $44 \%$.

These results are comparable to those reported by Allayali et al.(9) who reported that, fifty eight cases of psoriasis patients had plaque Psoriasis $(85.3 \%)$. They added, there was no significant difference between cases and control regarding comorbid condition, type of comorbid condition. However, only $4 \%$ in their work had positive family history, which is so lower than the present study.

Sacroiliac joint examination by X-ray revealed was normal in $76 \%$ of patients, showed blurring in $8 \%$, pseudowidening in $8.0 \%$, sclerosis in $4.0 \%$ and ankyloses in $4.0 \%$. Thus, the total rate of abnormaltiies was $24 \%$ and this sligtly higher than that reproted by Baskan et al. ${ }^{(8)}$ in psoriatic arthritis patients and reproted that, the vertebral deformity ratio was $19.2 \%$ in the PsA group, however, they included all deformities in both thoracic, lumber and sacroiliac regions and this can explain their increased rate of deformities when compared to the present work.

Conversely, Ibrahim et al. ${ }^{(6)}$ reported that, in X-Ray sacroiliac joint, they found $23.3 \%$ of patients have blurring of sacroiliac joint, $16.7 \%$ of patients have Pseudowidnening, $13.3 \%$ of patients with sclorsis of sacroiliac joint and $10 \%$ of patients have Ankylosis of joint. These data reflected high percentages of abnromalities in their study.
As regard to vitamin $\mathrm{D}$, it was sufficient in $32.0 \%$, insufficient in $34.0 \%$ and deficient in $34.0 \%$; and there was no significant difference between psoriasis and PA groups. In addition, vitamin D levels were increased in psoriasis when compared to PA groups. However, the difference was statistically non-significant. These results are comparable to previous work showed lower vitamin D levels to be found in patients with psoriasis and psoriatic arthritis Touma et al. ${ }^{(10)}$; Gisondi et al. ${ }^{(11)}$.

Zuchi et al. ${ }^{(12)}$ found that only 2 cases and 4 controls presented sufficient levels of vitamin D. Including all of the participants in the study, the median vitamin D level was $22.80 \pm 4.60$ and it was lower in those that were exposed to sunlight for less than 30 minutes per day.

In addition, a study with 121 patients who had inflammatory joint disease (22 psoriatic arthritis) revealed that, the levels of $25(\mathrm{OH})-\mathrm{D} 3$ in the patient group was lower than the levels observed in the control group Braun et al. ${ }^{(7)}$.

Furthermore, Braun and his collages ${ }^{(7)}$ found a high incidence of vitamin D deficiency in their patients. Also, Ibrahim et al. ${ }^{(6)}$ found a significant Vitamin D deficiency in psoriasis patients (skin psoriasis) (52.6) and insufficiency in (9.1\%) and normal in $(18.2 \%)$ while in the study done by Molina and his collages in 2012 they found significant Vitamin D deficiency in psoriatic arthritis.

In the present study, there was no signficant differnce between psoriasis and psoriatic arthritis as regard to serum vitmain D levels. However, there was signficant positve correlation between vitamin $\mathrm{D}$ levels and each of hemoglobin and RBCs, and there was inverse correlation between vitmain $\mathrm{D}$ and each of disease duration and disease severity (PASI score).

These results are comparable to those reported by Morimoto et al. ${ }^{(13)}$ who found an inverse relationship between the severity of psoriasis and $1,25[\mathrm{OH}] 2 \mathrm{D}$ levels in 34 patients. In addition, Ricceri et al.(14) found that $68 \%$ of psoriasis patients were $25[\mathrm{OH}] \mathrm{D}$ deficient. They reported a negative correlation with psoriasis area severity index (PASI).

Also, Chandrashekar et al. ${ }^{(15)}$ assessed the levels of $25[\mathrm{OH}] \mathrm{D}$, in patients with psoriasis versus controls. The PASI correlated negatively with 25[OH]D levels, as found in the present study.

Kincse et al. ${ }^{(16)}$ analysed 25[OH]D levels in 72 patients with psoriasis and psoriatic arthritis. An 
inverse correlation between $25[\mathrm{OH}] \mathrm{D}$ level and skin severity as well as severity of psoriatic arthritis was identified. However, in the present study, no signficant correlation was found between serum levels of vitmain $\mathrm{D}$ and psoriatic arthritis severity indices.

In addition, Osmancevic et $\boldsymbol{a l}^{\left({ }^{(17)}\right.}$ found that the PASI score correlated negatively with serum level of $25(\mathrm{OH})$ vitamin $\mathrm{D}$.

On the other hand, Atwa et $\boldsymbol{a l} .^{(18)}$ reported that, 25[OH]D levels did not correlate with psoriasis severity as assessed by PASI. This results are in contradiction to the present study and may be explaiend by different inclusion criteria.

Vitamin D plays a critical role in psoriasis, and this is evidenced in many studies which reported either a deficiency or insufficiency of serum vitamin D in psoriatic patients Mattozzi et al. $^{(19)}$; Maleki et al. ${ }^{(20)}$.

Several case-control studies have shown significant lower levels of serum $25(\mathrm{OH}) \mathrm{D}$ in psoriatic patients compared to controls and reported an inverse correlation between serum $25(\mathrm{OH}) \mathrm{D}$ and the severity of the disease ${ }^{(15,21,22)}$. These results are comparable to that of the present study.

On the other hand, in a population-based screening, Wilson ${ }^{(23)}$ showed that vitamin D deficiency is not common in psoriatic patients and that there is no significant difference in serum $25(\mathrm{OH}) \mathrm{D}$ levels in subjects with or without psoriasis. This can be explaied by the fact that, the $25(\mathrm{OH}) \mathrm{D}$ level varies with several factors, including race, dietary intake, and UV light exposure. In addition, vitamin D insufficiency is a common finding in normal populations.

In the present work, there was no significant relation between vitamin $\mathrm{D}$ levels and each of sex, residence, smoking, hypertension and diabetes. In addition, there was no significant relation between vitamin $\mathrm{D}$ and disease onset type, course, family history or nail affection. Furthermore, there was no sigficant correlation between vitamin D and age, BMI, WBCs, platelets, urea, creatinine, SGOT, SGPT and fasting blood glucose. These resutls are in agreement with Ibrahim et $\boldsymbol{a l}^{\left({ }^{(6)}\right.}$ who reported that, there was no significant statistical difference between vitamin D levels in relation to sex, and Braun et al $^{(7)}$ who reported that, there was no significant statistically correlations between $25(\mathrm{OH}) \mathrm{D}$ and gender, type of disease, season (winter and summer) and type of treatment. Older patients (age >60 years) had lower levels of $25(\mathrm{OH}) \mathrm{D}$, but the correlation was of not signficant.
In the present work, patients with elevated ESR had a low levels of serum vitmain D when compared to cases with normal ESR (23.94 \pm 7.47 vs $25.08 \pm 7.43$ respectivley). However, the differnce was statistically non signficant and thus there was no signficant correlation between vitamin D and ESR. These results are in contradiction in those reported by Ibrahim et $\boldsymbol{a l} .{ }^{(6)}$ who reported that, $70 \%$ of their patients with elevated ESR have deficiency in vitamin D level, $15 \%$ of patients have insufficiency and $15 \%$ of patients have normal vitamin $\mathrm{D}$ level. However, results of the present wrok go in agreement with Braun et al. ${ }^{(7)}$ They found that no significant statistically correlations between vitamin D level, in relation to ESR.

In the present work, patients with elevated CRP had lower levels of vitamin D when comapred to patients with normal CRP $(23.80 \pm 7.68$ vs $25.57 \pm 6.98$ respectivley). However, the differnce was statistically non-signficant. These results are in accordance with Molina et $\boldsymbol{a l} .{ }^{(24)}$ and Ibrahim et $\boldsymbol{a l}{ }^{(6)}$ who reorted that, there was no significant statistical difference between vitamin D levels in relation to CRP and there was no correlation between vitamin D levels in relation to CRP.

\section{CONCLUSION}

Vitamin D deficiency/insufficiency was common among psoriasis and psoriatic arthritis patients and the dificnecy was signficnatly and inversly correlated with disease severity (PASI) and disease duration. Thus, is though to play a role in development (or a consequence) of psoriasis. On the other hand, there was no corrlation with PA disease activity and severity indices. Thus, the association seems to be linked to psoriais proper.Vitamin D supplementation thought to have a potential therapeutic role in treatment of psoriasis.

\section{REFERENCES}

1. Mrowietz U, Reich K (2009): Psoriasis - New Insights Into Pathogenesis and Treatment. Dtsch Arztebl Int., 106(1-2): 11-9.

2. Shlyankevich J, Mehta $\mathrm{N}$, Krueger $\mathrm{J}$ et al. (2014): Accumulating evidence for the association and shared pathogenic mechanisms between psoriasis and cardiovascularrelated co-morbidities. Am J Med., 127: 1148-1153. 
3. Mostafa WZ and Hegazy RA (2015): Vitamin $\mathrm{D}$ and the skin: Focus on a complex relationship: A review. J Adv Res., 6 (6): 793-804.

4. Moroni L, Bianchi I and Lleo A (2012): Geoepidemiology, gender and autoimmune disease. Autoimmun Rev., 11: 386-392.

5. Zold E, Barta Z, and Bodolay E (2011): Vitamin D deficiency and connective tissue disease. Vitam Horm, 86: 261-286.

6. Ibrahim AM, Altamimy HM, Moneer M, Abdul-Hamied HI (2013): Measurement of vitamin (d) and its relation to psoriatic arthritis patiants. AAMJ., 11 (2):292-304.

7. Braun M, Toledano K, Markovits D, Rozin A et al. (2011): Vitamin D level: is it related to disease activity in inflammatory joint disease? Rheumatol Int, 31:493-499.

8. Baskan B, Oten E, Sivas F, Eser F, Yurdakul FG, Duran S, Bodur H (2016): The relationship between vitamin $\mathrm{D}$, vertebral deformity and quality of life in psoriatic arthritis. Acta Reumatol Port.,41:350-358

9. Allayali A, Niaz G, Hawsawi KA, Fatani M, Siddiqui I et al. (2018): Association between Vitamin D Deficiency and Psoriasis: A CaseControl Study. J Clin Exp Dermatol Res., 9: 442.

10. Touma Z1, Eder L, Zisman D, Feld J, Chandran V, Rosen CF, Shen H, Cook RJ, Gladman DD (2011): Seasonal variation in vitamin D levels in psoriatic arthritis patients from different latitudes and its association with clinical outcomes. Arthritis Care Res (Hoboken),63:1440-1447.

11. Gisondi P1, Rossini M, Di Cesare A, Idolazzi L, Farina S, Beltrami G, Peris K, Girolomoni G (2012): Vitamin D status in patients with chronic plaque psoriasis. $\mathrm{Br} \mathrm{J}$ Dermatol., 166:505--510.

12. Zuchi M, Azevedo $P$, Tanaka A et al. (2015): Serum levels of 25-hydroxy vitamin D in psoriatic patients, 90(3): 430-432.

13. Morimoto S, Yoshikawa $K$, Fukuo $K$, Shiraishi T, Koh E, Imanaka S, Kitano S, Ogihara $T$ (1990): Inverse relation between severity of psoriasis and serum 1,25-dihydroxyvitamin D level. J Dermatol Sci., 1:277-282

14. Ricceri F, Pescitelli L, Tripo L, Prignano F (2013): Deficiency of serum concentration of 25hydroxyvitamin D correlates with severity of disease in psoriasis. J Am Acad Dermatol., 68:511-512.
15. Chandrashekar L, Kumarit GR, Rajappa M, Revathy G, Munisamy M, Thappa DM (2015): 25-hydroxy vitamin D and ischaemiamodified albumin levels in psoriasis and their association with disease severity. $\mathrm{Br} \mathrm{J}$ Biomed Sci., 72:56-60

16. Kincse G, Bhattoa PH, Heredi E, Varga J, Szegedi A, Keri J, Gaal J (2015): Vitamin D3 levels and bone mineral density in patients with psoriasis and/or psoriatic arthritis. J Dermatol., 42:679-684.

17. Osmancevic A, Landin-Wilhelmsen K, Larkö O, Krogstad AL(2010): Vitamin D status in psoriasis patients during different treatments with phototherapy, 101(2):117-23.

18. Atwa MA, Balata MG, Hussein AM, Abdelrahman NI, Elminshawy HH (2013): Serum 25-hydroxyvitamin D concentration in patients with psoriasis and rheumatoid arthritis and its association with disease activity and serum tumor necrosis factor-alpha. Saudi Med J., 34:806-813.

19. Mattozzi C, Paolino G, Salvi M, Macaluso L, Luci C, Morrone S, Calvieri S, Richetta AG (2016): Peripheral blood regulatory $T$ cell measurements correlate with serum vitamin $\mathrm{D}$ level in patients with psoriasis. Eur Rev Med Pharmacol Sci., 20: 1675-1679.

20. Maleki M, Nahidi Y, Azizahari S, Meibodi NT, Hadianfar A (2016): Serum 25-OH vitamin $\mathrm{D}$ level in psoriatic patients and comparison with control subjects. J Cutan Med Surg., 20: 207-210.

21. Orgaz-Molina J, Magro-Checa C, RosalesAlexander JL, Arrabal-Polo MA, CastelloteCaballero L, Buendia-Eisman A, RayaAlvarez E, Arias-Santiago S (2014): Vitamin $\mathrm{D}$ insufficiency is associated with higher carotid intima-media thickness in psoriatic patients. Eur J Dermatol., 24: 53-62.

22. Maruotti N, Cantatore FP. (2010): Vitamin $\mathrm{D}$ and the immune system. The Journal of Rheumatology, 37, 491-495.

23. Wilson PB (2013): Serum 25-hydroxyvitamin D status in individuals with psoriasis in the general population. Endocrine, 44: 537-539.

24. Molina J, Buendía EA, Arrabal-Polo MA, Ruiz JC, Arias-Santiago S (2012): Deficiency of serum concentration of 25-hydroxyvitamin D in psoriatic patients: a case-control study.J Am Acad Dermatol.,67(5):931-8. 